\title{
Percutaneous Transluminal Coronary Angioplasty for Postoperative Left Coronary Artery Stenosis Following Surgical Reconstruction of Congenital Atresia of the Left Main Coronary Artery
}

\author{
Daiji Takeuchi; Yoshiki Mori; Kanta Kishi; Tae Nakajima; Makoto Nakazawa; \\ Yukio Tsurumi*; Hiromi Kurosawa**; Toshio Nakanishi
}

\begin{abstract}
Congenital atresia of the left main coronary ostium is a rare coronary artery anomaly. A 3-year-old boy who was asymptomatic had a heart murmur because of mitral regurgitation. He underwent reconstruction of the left main coronary artery, but stenosis occurred in the early postoperative period. Although the patient underwent repair of the coronary artery stenosis, the distal portion of the left coronary artery re-stenosed. Percutaneous transluminal coronary angioplasty for the stenosis was performed successfully and there has not been any sign of re-stenosis for 30 months to date. (Circ J 2009; 73: 2360-2362)
\end{abstract}

Key Words: Atresia; Coronary artery stenosis; Left main coronary ostium; Percutaneous transluminal coronary angioplasty

$\mathbf{C}$ ongenital atresia of the left main coronary ostium (LMCA) is a rare condition in which there is no left coronary ostium and the proximal main trunk ends blindly. ${ }^{1-3}$ Among the different surgical approaches, including various forms of coronary artery bypass (eg, using the saphenous vein or internal mammary artery), reconstruction is the current technique of choice. However, postoperative coronary artery stenosis is a major complication. Although percutaneous transluminal coronary angioplasty (PTCA) is an acceptable treatment for adult patients with coronary artery stenosis, experience with postoperative coronary stenosis in pediatric patients remains limited. ${ }^{4-6}$ We report the mid-term result of PTCA in a 3-year-old boy with postoperative left coronary artery stenosis following surgical reconstruction of the LMCA.

\section{Case Report}

A boy was referred to hospital at 2.5 years of age for assessment of heart murmur. On physical examination, a grade 3 systolic murmur and a $3^{\text {rd }}$ heart sound were heard in the mitral area. Chest X-ray showed cardiomegaly with left atrial enlargement. Resting ECG demonstrated left atrial enlargement, but there were no ST-T changes. Echocardiography demonstrated a dilated left ventricle and atrium, with severe mitral regurgitation (MR). Myocardial thallium (Tl) scan concomitant with ${ }^{123}$ I-BMIPP showed diffuse dissociation between perfusion and lipid metabolism, consistent with ischemia of the left main coronary artery perfusion area. At cardiac catheterization, left ventricular angiography showed a normal ejection fraction of $71 \%$ and dilation of the end-diastolic volume was $387 \%$ of normal, with grade $4 \mathrm{MR}$. On selective right coronary angiography, the right coronary artery was dilated with retrograde filling of the left coronary artery via collaterals, but there was no passage of contrast media from the left coronary artery to the main

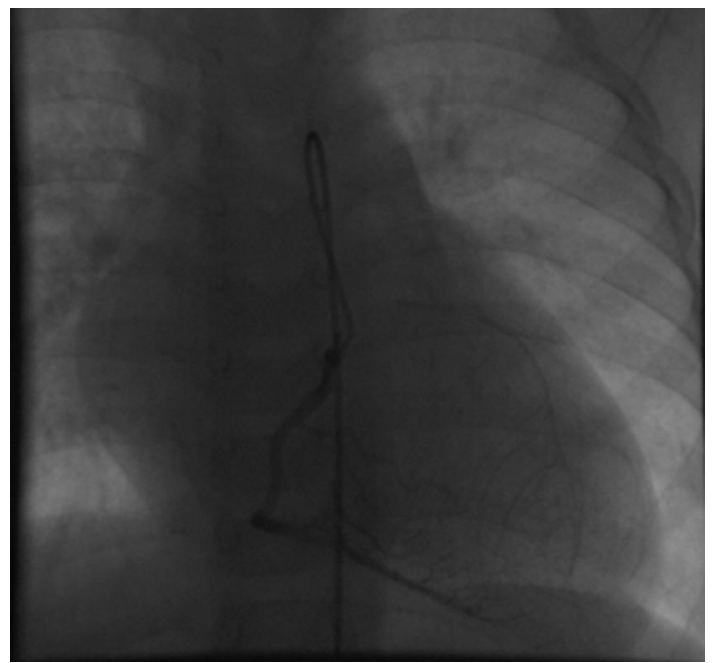

Figure 1. Selective right coronary angiography shows dilated right coronary artery with retrograde filling of the left coronary artery via collaterals.

Received April 24, 2008; revised manuscript received March 22, 2009; accepted April 16, 2009; released online June 3, 2009

Departments of Pediatric Cardiology, *Cardiology and **Cardiovascular Surgery, Heart Institute, Tokyo Women's Medical University, Tokyo, Japan

Mailing address: Daiji Takeuchi, MD, Department of Pediatric Cardiology, Heart Institute, Tokyo Women's Medical University, 1-8 Kawadacho, Shinjuku-ku, Tokyo 161-8666, Japan. E-mail: daiji-takeuchi@nifty.com

All rights are reserved to the Japanese Circulation Society. For permissions, please e-mail: cj@j-circ.or.jp 
A

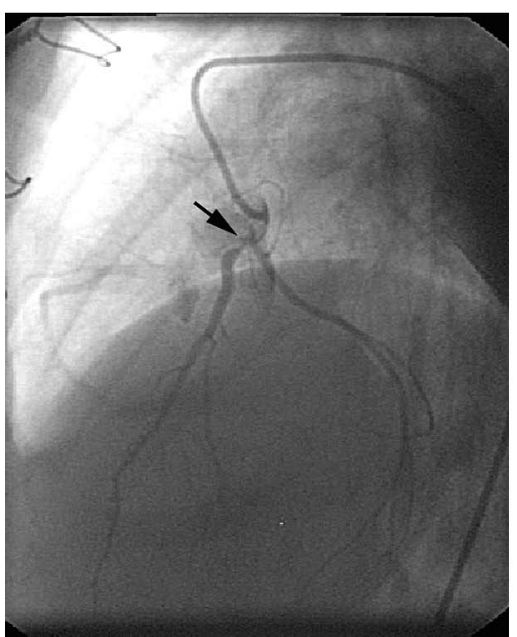

B

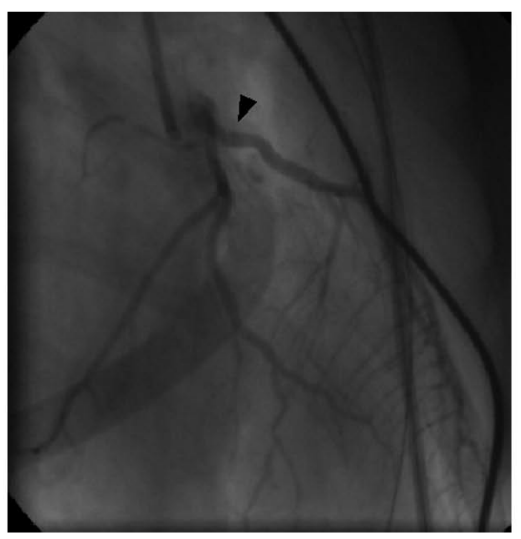

C

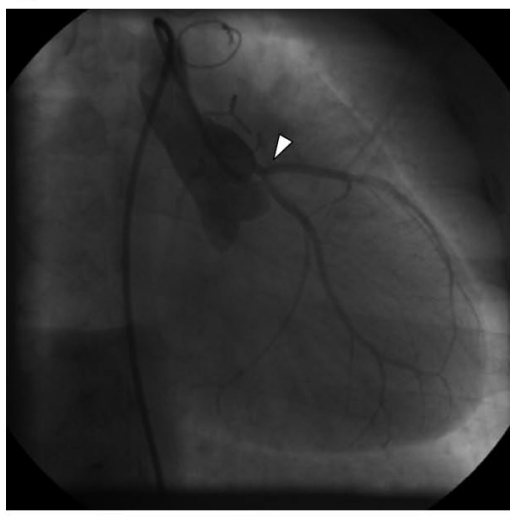

Figure 2. (A) Selective left coronary angiography shows a localized $90 \%$ stenosis of the left anterior descending artery (proximal portion of segment 6) (arrow). (B) Stenotic lesion dilated from $90 \%$ to $25 \%$ stenosis by percutaneous transluminal coronary angioplasty (arrowhead). (C) Follow-up coronary angiogram 30 months after percutaneous transluminal coronary angioplasty does not show any significant progression of stenosis (white arrowhead).

pulmonary artery or ascending aorta (Figure 1). The left coronary artery could not be selectively catheterized from the aortic root.

The patient underwent surgery at 2.7 years of age. Direct observation through aortotomy showed that there was no left coronary ostium in the usual position, although a blind dimple was seen on top of the commissure between the left and noncoronary cusps. The left coronary artery was anastomosed to the aorta and then enlarged using the azygos vein. In brief, the aortotomy was extended toward the left coronary cusp. The left main trunk climbing straight up along the ascending aorta was recognized and cut to $4 \mathrm{~mm}$ in diameter at the middle. The proximal portion of the left main trunk was recognized using a 1-mm probe from the incision to just before the aorta wall, but it had no connection to the ascending aorta. Part of the left main trunk incision was anastomosed with the lower margin of the aortotomy and the remaining part was applied using the azygos vein. Mitral valve plasty was also performed.

Postoperatively, the patient had an uneventful recovery, but 6 months later Tl-myocardial scintigraphy using dipyridamole-infusion showed diffuse ischemia. Postoperative cardiac catheterization showed severe stenosis in the proximal portion of the left main coronary artery (segment 5), although collaterals from the right coronary artery had disappeared. Therefore, re-operation with left coronary ostium plasty using autologous pericardium was performed 7 months after the first procedure. In brief, transverse aortotomy demonstrated localized stenosis at the distal end of the suture line of the previously reconstructed left main trunk using azygos vein. Therefore, the aortotomy was extended to the left circumflex artery beyond the bifurcation and the remaining part of the aortotomy received a triangular autologous pericardial patch to relieve the coronary artery stenosis.

However, follow-up Tl myocardial scintigraphy using dipyridamole-infusion showed ischemic change of the left ventricular anterior wall and there was ST-T depression in the $\mathrm{V}_{4-6}$ leads on ECG. The cardiac catheterization showed localized $90 \%$ stenosis of the left anterior descending artery (distal portion of the reconstructed segment 6) at 3.5 years of age (Figure 2A). The diameter of the left internal thoracic artery was determined on angiography and considered too small to be used as a coronary bypass graft. The saphenous vein was also too small and inadequate for grafting. Therefore, PTCA was scheduled for the management of coronary artery stenosis and was performed after artificial pulmonary circulatory support was set up with thoracotomy. A 5-Fr left Judkins catheter was positioned in close proximity to the stenotic lesion and a 0.014-inch exchange guidewire was advanced through the catheter into the stenotic coronary artery. The stenotic segment was dilated using a $2.0-\mathrm{mm}$ balloon catheter (VENTO, Invatec s.c.l, Italy), which was changed to a 2.75-mm balloon catheter (Q-Maverick, Boston Scientific, Natick, MA, USA). The degree of stenosis was improved from $90 \%$ to $25 \%$ (Figure 2B).

Follow-up coronary angiography was performed 30 months after PTCA and did not show any significant progression of stenosis (Figure 2C). ${ }^{201} \mathrm{Tl}$ myocardial imaging using dipyridamole infusion did not show any significant ischemic findings in the anteroseptal wall of the left ventricle.

\section{Discussion}

The incidence of congenital anomaly of the coronary artery is estimated to range from $0.2 \%$ to $1.2 \%$ of the general population. ${ }^{7}$ Among the congenital anomalies of the coronary artery, LMCA is extremely rare with less than 50 cases reported in the literature. ${ }^{2,3} \mathrm{~A}$ definitive diagnosis of LMCA is established by an invasive method or intraoperatively, and noninvasive diagnosis is difficult. $2,3,8,9$ In this case, the first sign was heart murmur because of MR, which could have been the result of papillary muscle dysfunction subsequent to myocardial ischemia. This rare condition should be considered in pediatric patients with significant MR. The LMCA carries an unfavorable prognosis, such as sudden death, which medical treatment does not seem to prevent. Surgical revascularization is considered the best treatment for both symptomatic and asymptomatic patients with LMCA. After reviewing the literature describing 28 cases 
of LMCA, Musiani et al reported that the outcome of the patients who did not receive surgical therapy was poor. ${ }^{2}$ The surgical treatment for LMCA has not been established. Although coronary artery bypass was the procedure of choice in many cases reported previously, 1,2,8,9 long-term patency, especially when using the saphenous vein in pediatric patients, was questionable. Therefore, we selected reconstruction of the left main coronary artery. The 2-coronary artery system was expected to restore adequate coronary blood flow to the ischemic myocardium, independently of retrograde collateral flow from the right coronary artery, by reconstruction of the left main coronary artery. Recently, Varghese et al reported a similar case in which the left main coronary artery was reconstructed with autologous pericardial patches; however, the mid- and long-term results of that procedure remain unclear. ${ }^{3}$ In the present case, left coronary artery stenosis occurred in the very early postoperative period and stenosis of a different portion of the left coronary artery developed after repairing the proximal left main coronary artery. The reasons for coronary stenosis after surgical repair could not be clearly detailed, because we did not perform intravascular ultrasound to obtain information about vascular morphology just after the surgical repair. Possible mechanisms involved in postoperative coronary artery stenosis would include excessive scar formation or abnormal intimal proliferation, in addition to thrombus formation. We performed balloon dilatation rather than stent implantation, expecting future growth of the coronary artery. Recently, Kampmann et al demonstrated the long-term efficacy of PTCA in 7 children with proximal coronary stenoses after an arterial switch procedure for d-transposition of the great arteries. ${ }^{6}$ In the present case, the balloon to poststenotic artery ratio was more than 1.0. It has been reported that there is no residual stenosis when PTCA has been performed with a balloon to post-stenotic artery ratio between 1.0 and 1.4 after arterial switch procedure. ${ }^{5}$ It may be necessary to perform PTCA using an oversized balloon to dilate the scar lesions in postoperative patients. Thus, PTCA for postoperative coronary artery stenosis differs from PTCA for atherosclerotic lesions, because overexpansion of a vessel containing an atherosclerotic lesion is considered to promote a higher degree of restenosis. PTCA using an oversized balloon is an option to relieve postoperative coronary stenosis in growing children. This is the first report showing the mid-term efficacy of PTCA for coronary artery stenosis after repair of LMCA atresia.

\section{References}

1. Mullins C, El-Saido G, McNamara DG, Cooley DA, Treistman B, Garcia E. Atresia of the left coronary artery ostium: Repair by saphenous vein graft. Circulation 1972; 46: 989-994.

2. Musiani A, Cernigliaro C, Sansa M, Maselli D, De Gasperis C. Left main coronary artery atresia: Literature review and therapeutical considerations. Eur J Cardiothorac Surg 1997; 11: 505-514.

3. Varghese P, Leanage RU, Peek GJ. Congenital atresia of the ostium of the main coronary artery: A rare coronary anomaly, diagnostic difficulty and successful surgical revascularization. Congenital Heart Dis 2007; 2: 347-350.

4. Shaddy RE, Revenaugh JA, Orsmond G, Tani LY. Coronary interventional procedures in pediatric heart transplant recipients with cardiac allograft vasculopathy. Am J Cardiol 2000; 85: 1370-1372.

5. De Caro E, Pongiglion G. Percutaneous transluminal angioplasty of the left internal thoracic artery graft: A case report of a child operated on for anomalous origin of the left coronary artery from the pulmonary artery. Pediatr Cardiol 2001; 22: 423-425.

6. Kampmann C, Kuroczynski W, Trubel H, Knuf M, Schneider M, Heinemann MK. Late results after PTCA for coronary stenosis after the arterial switch procedure for transposition of the great arteries. Ann Thorac Surg 2005; 80: 1641-1646.

7. Garg N, Tewari S, Kapoor A, Gupta DK, Sinha N. Primary congenital anomalies of the coronary arteries: A coronary arteriographic study. Int J Cardiol 2000; 74: 39-46.

8. Gerlis LM, Magee AG, Sheppard MN. Congenital atresia of the orifice of the left coronary artery. Cardiol Young 2002; 12: 57-62.

9. Sunagawa M, Shimabuluro T, Kado H, Ushinohama H, Ohta T. Congenital atresia of the left main coronary artery; successful surgical treatment (myocardial revascularization and mitral valve repair) in a 1-year-old boy. Eur J Pediatr 2005; 164: 461 - 462. 